\title{
Recognition of Tumoursin Human Cerebrum
}

\author{
J.Deny $^{1}$, R. Raja Sudharsan ${ }^{2}$ \\ \{prof.j.deny@gmail.com ${ }^{1}$, rajasudharsan5255@gmail.com² ${ }^{2}$
}

Department of Electronics and Communication Engineering, Kalasalingam Academy of Research and Education, Krishnan Koil, Virudhunagar, 626126, India. ${ }^{1,2}$.

\begin{abstract}
One of the difficult errands in the medicinal field is cerebrum tumour order which includes the extraction of tumour districts from pictures. By and large, this undertaking is being done physically by medicinal specialists which isn't constantly evident because of the similitude among tumour and ordinary tissues and the high decent variety in tumours' appearance. Accordingly, computerizing restorative picture division stays a genuine test. In this paper, we will concentrate on bunching of Magnetic Resonance cerebrum Images (MRI) by utilization of k-Nearest Neighbours calculation. Our thought is to consider this issue as a grouping issue where the point is to recognize ordinary and anomalous pixels based on a few highlights, in particular forces and surface. All the more decisively, it is recommended to utilize SVM which is mainstream and spurring characterization techniques. The exploratory investigation is experimented for Gliomas dataset speaking to various tumour shapes, areas, sizes and picture powers and furthermore to recognize blood clusters in the human mind.
\end{abstract}

Keywords: Support Vector Machine(SVM), Brain tumour, Brain tumour Segmentation, Gliomas, Magnetic Resonance Imaging

\section{Introduction}

The most widely recognized essential mind tumour is meningioma with $34 \%$, anyway glioma, an expansive term including tumours emerging from the gluey or strong tissue of the cerebrum (30\% of all mind tumours), speaks to $80 \%$ of harmful tumours making it the most well-known essential mind tumour causing passing [1]. The most widely recognized and forceful glioma is glioblastoma various (GBM) speaking to $54 \%$ all things considered [4]. This kind of tumour is joined by quick infiltrative development and poor visualization with one-year normal endurance time after analysis. The endurance time is influenced by broad treatment, for example, chemo-and radiotherapy and careful resection. This work is especially centred on the programmed handling of volumes with the most widely recognized dangerous tumour - glioma - in low and high evaluations [2].

The manner clinical schedules, the assessment of procured pictures are presently performed physically dependent on quantitative criteria or measures, for example, the biggest unmistakable width in pivotal cut [3]. Hence, profoundly exact techniques having the option to consequently examine sweeps of cerebrum tumor would have a colossal potential for determination and treatment arranging [5]. In any case, it was demonstrated that even manual explanation performed by master raters indicated huge varieties in territories where force inclinations between tumorous structures and encompassing tissues are smooth or clouded by predisposition field relics or the halfway volume impact. Additionally, cerebrum tumor sores are just characterized by relative force changes to solid tissues, and their shape, size and area 
are individual for every patient, which utilizes regular example acknowledgment calculations unthinkable.

\section{Methodology}

The fig.1. represents the overview of this proposed technique. There are three main stages: Pre-processing, Segmentation and Classification.

\section{(i) Pre-Processing:}

Pre-preparing is the name for exercises on pictures at the most decreased level of reflection. It doesn't expand picture data content. Image pre-processing utilizes the repetition in pictures.

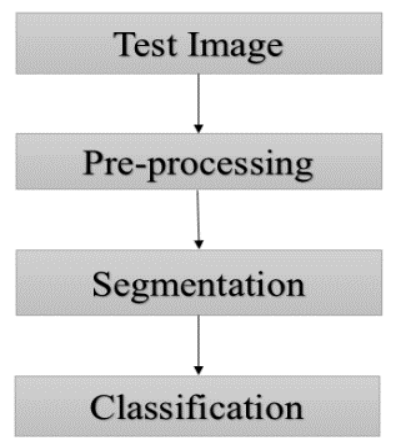

Fig 1: Overview of Proposed Method

In pre-processing, middle filtering system is utilized. The centre channel is a nonlinear modernized isolating system, as often as possible used to remove fuss from an image or sign. Such disturbance decline is a typical pre-preparing dare to improve the eventual outcomes of later taking care of. Centre filtering is commonly used in modernized picture dealing with because, under explicit conditions, it jam edges while removing clamour.

Centre Filtering is a nonlinear technique used to expel clamour from images. It is especially powerful at expelling black and white type commotion [6]. The centre channel works by going through the image pixel by pixel, superseding each a motivating force with the centre advantage of neighbouring pixels.

Edges are of fundamental essentialness to the visual appearance of pictures, for example. For little to coordinate degrees of clatter, the centre channel is irrefutably better than Gaussian fog at clearing uproar while defending edges for ensured, fixed window size. In any case, its presentation isn't that greatly improved than Gaussian haze for elevated levels of clamour, while, for dot commotion and rash clamour, it is especially powerful. Along these lines, middle sifting is broadly utilized in computerized picture preparing.

\section{(ii) Clustering}

Grouping (Clustering) is done to separate among ordinary and anomalous in the human cerebrum. k-Nearest Neighbours calculation is utilized for bunching. The objective of this grouping strategy is to just separate the information dependent on the expected similitudes between different classes.

k-Nearest Neighbours is a fundamental portrayal computation. It has a spot with the coordinated learning region and finds remarkable application in plan affirmation, data mining and interference disclosure. It is commonly superfluous, in fact, circumstances since it is non- 
parametric, which implies, it doesn't make any fundamental doubts about the scattering of data. The test name is gotten from its k-closest neighbours from the preparation set in the component space.

It is hard to precisely separate a halfway influenced tissue. By utilization of k-Nearest Neighbours it is conceivable to amass the estimated qualities

It is difficult to accurately differentiate a partially affected tissue. By use of k-Nearest Neighbours it is possible to group the approximate values.

\section{(iii) Segmentation}

Picture (Image) Segmentation is the route toward allocating a modernized picture into various pieces. Picture division is ordinarily used to discover things and cut off points (lines, twists, etc.) in pictures. The eventual outcome of depiction dissection is a ton of parts that all things considered spread the complete representation, or a great deal of erections expelled from image (see edge distinguishing proof). Bordering regions are on a very basic level one of a kind concerning comparative characteristics. In this endeavor picture is divided using reinforce vector machine calculation. "Bolster Vector Machine" is a managed machine learning estimation which can be used for both gathering or backslide challenges. Nevertheless, it is generally used in gathering issues. In this count, we plot each datum thing as a point in n-dimensional space with the estimation of every segment being the estimation of a particular composes. Bolster Vectors are essentially the co-ordinates of individual discernment. SVM is a wild which best detaches the two classes.

Linear SVM is the most current amazingly quick AI calculation for tackling multiclass order issues from ultra-huge informational collections that executes a unique exclusive variant of a cutting plane calculation for structuring a direct help vector machine. Straight SVM is a directly adaptable routine implying that it makes a SVM model in a CPU time which scales directly with the size of the preparation informational collection. Our correlations with other known SVM models plainly demonstrate its prevalent exhibition when high exactness is required.

\section{Experimental Setup and Result Analysis}

All MRI data was provided by the 2015 MICCAI BraTS Challenge, which consists of approximately 250 high-grade glioma cases and 50 low-grade cases. The MRI of brain is segmented and classified using Support Vector Machine with 4 kernel functions.RBF accuracy, Linear accuracy, Polygonal accuracy, Quadratic accuracy are calculated in the following images.

Role of Kernel Functions in SVM: To select a proper classification algorithm for image is really a tedious task. The linear SVM classifier takes an input data from feature set and classifies into two possible classes. That's why the nonlinear SVM works in a good way on high dimensional feature sets. To increase the margin of the classification, kernel functions are used. There are many kernels used in support vector machine such as Radial Basis Function (RBF), linear, polynomial etc. Kernel SVM gives the clear understanding of the classification and very easy to use in practical image processing. In this paper some of its methods like RBF, linear, and polygonal are used to find the segmented image. 


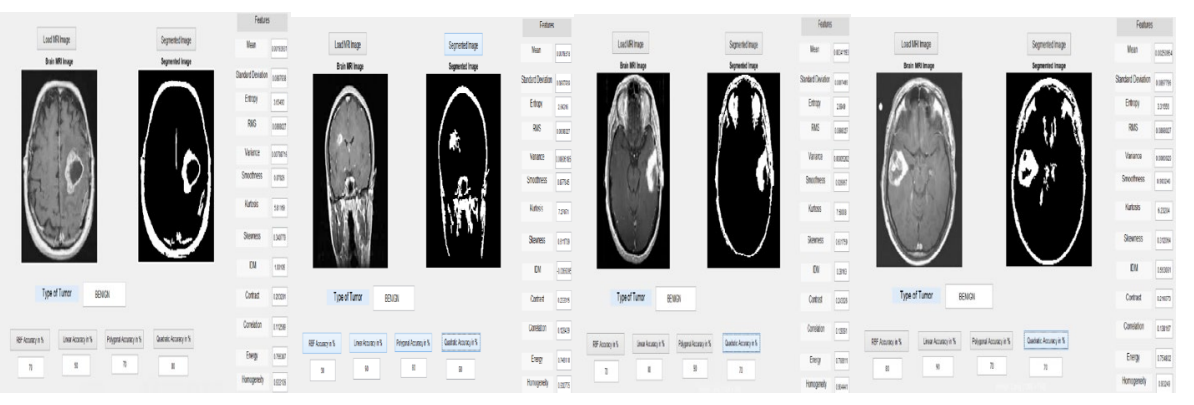

(a)

(b)

(c)

(d)

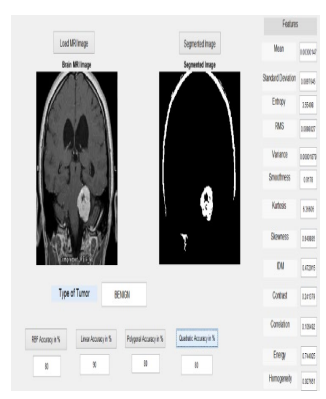

(e)

Fig. 2. Segmented Images of different Benign Tumor from Dataset

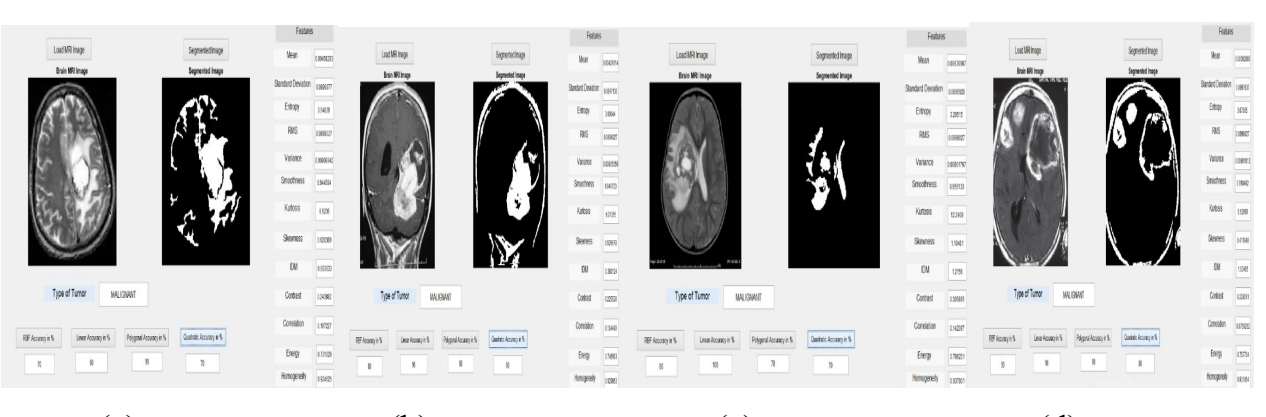

(a)

(b)

(c)

(d) 


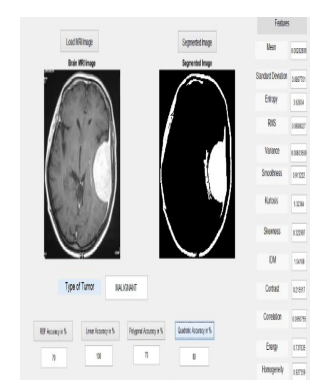

(e)

Fig. 3. Segmented Images of different Malignant Tumour from Dataset

\section{Conclusion}

The MRI of brain is segmented and classified using Support Vector Machine. In particular, we have analysed and compared four of the kernel functions of SVM with a standard brain MRI data. In future we can use others feature reduction method and can compare and find the accuracy. The researcher can also use various hybridized learning algorithms with variety of datasets.

\section{References}

[1] Sudharsan, RR, J. Deny, E. Muthu Kumaran, and A. Sharon Geege. "An Analysis of Different Biopotential Electrodes Used for Electromyography", 2020.

[2] Ed-EdilyMohd. Azhari1, Muhd. MudzakkirMohd. Hatta1, ZawZawHtike, "Brain Tumor Detection and Localization in Magnetic Resonance Imaging", International Journal of Information Technology Convergence and Services (IJITCS) DOI:10.5121/ijitcs.2014.4101

[3] Sudharsan, R.R., Deny, J., Muthukumaran, E. et al. FPGA based peripheral myopathy monitoring using MFCV at dynamic contractions. J Ambient Intell Human Comput (2020). https://doi.org/10.1007/s12652-020-02363-7

[4] Sudharsan, RR, and J. Deny. "Field Programmable Gate Array (FPGA)-Based Fast and Low-Pass Finite Impulse Response (FIR) Filter" In Intelligent Computing and Innovation on Data Science, pp. 199-206. Springer, Singapore, 2020.

[5] Deny, J., and Sudharsan, RR. "Block Rearrangements and TSVs for a Standard Cell 3D IC Placement." In Intelligent Computing and Innovation on Data Science, pp. 207-214. Springer, Singapore, 2020.

[6] J.Deny ,N.Sivasankari, "Biometric Security in Military Application ” Journal of Engineering Procedia ELSEVIER,Vol.38,pp-1138-1144.

[7] J.Deny, Dr.M.Sundhararajan"Survey of Texture Analysis Using Histogram in Image Processing" International Journal of Applied Engineering Research (IJAER) Vol 26, pp .8737-8739.

[8] J.Deny, Dr.M.Sundhararajan "Review of Color Image Segmentation Using K-means and SuperPixel Algorithm" International Journal of Digital Communication and Networks, Vol. 2, Issue 12, pp: 4-8.

[9] Dr.E.Muthukumaran. Dr.J.Deny, Dr.B.Perumal,G. Suseendran., Dr. D.Akila "A narrative NonInvasive Diagnostic loom Based by the side of correlation of Nasal set Rhythm in addition to customary Three Radial Pulses Measurement" IOP Conf. Series: Journal of Physics: Conf. Series 1228 (2019) 012075. 
[10] Deny John Samuvel, B. Perumal, Muthukumaran Elangovan, "Music recommendation system based on facial emotion recognition, Publicado en 3C Tecnología. Special Issue - March 2020. 